\title{
Dynamic phase transition features of the cylindrical nanowire driven by a propagating magnetic field
}

\author{
*Erol VATANSEVER(0000-0003-1222-3941) \\ Department of Physics, Faculty of Science, Dokuz Eylul University, Izmir, Turkey \\ erol.vatansever@deu.edu.tr
}

Arrival Date: 08.11.2017

Accepted Date: 31.05 .2018

\begin{abstract}
Magnetic response of the spin-1/2 cylindrical nanowire to the propagating magnetic field wave has been investigated by means of Monte Carlo simulation method based on Metropolis algorithm. The obtained microscopic spin configurations suggest that the studied system exhibits two types of dynamical phases depending on the considered values of system parameters: Coherent propagation of spin bands and spin-frozen or pinned phases, as in the case of the conventional bulk systems under the influence of a propagating magnetic field. By benefiting from the temperature dependencies of variances of dynamic order parameter, internal energy and the derivative of dynamic order parameter of the system, dynamic phase diagrams are also obtained in related planes for varying values of the wavelength of the propagating magnetic field. Our simulation results demonstrate that as the strength of the field amplitude is increased, the phase transition points tend to shift to the relatively lower temperature regions. Moreover, it has been observed that dynamic phase boundary line shrinks inward when the value of wavelength of the external field decreases.
\end{abstract}

Keywords: Cylindrical nanowire, propagating magnetic field, Monte Carlo simulation.

\section{INTRODUCTION}

Interacting spin systems driven by a sinusoidal oscillating magnetic field can exhibit distinctive and fascinating dynamic behaviours, which cannot occur for the corresponding equilibrium spin systems. For the first time in Ref. [1], the authors applied their theoretical model to investigate the physics underlying a simple ferromagnet being exposed to a time dependent magnetic field. From their analysis, the authors concluded that amplitude and period of the oscillatory magnetic field play an important role on the dynamic characters of the considered magnetic system. Since then, many theoretical [2-15] and several over a full cycle of the external field. In addition to the consensus in dynamic phase transitions and equilibrium phase transitions, however, there are inconsistencies in the literature, in view of the universality class of the spin systems. For instance, recent detailed Monte Carlo (MC) simulations studies show that there is a clear difference between critical dynamics of the magnetic system with surfaces and its equilibrium case [9]. From their finite-size scaling analysis, the authors found that nonequilibrium surface exponents do not coincide with those of the experimental works [16-20] have been carried to understand the origin of the dynamic phase transitions. Based on the some of the previously published studies, it is possible to say that there is a good consensus between dynamic phase transitions and equilibrium phase transitions. For example, it has been reported that the critical exponents of the two-dimensional kinetic Ising model subjected to a square-wave oscillatory magnetic field are consistent with the universality class of the corresponding equilibrium Ising model [5]. Moreover, it is recommended in these references [12, 15, 17, and 19] that bias field appears to be a conjugate field of the dynamic order parameter, which is time averaged magnetization

equilibrium critical surface. Very recently, it is reported both experimentally and theoretically that there are metamagnetic fluctuations in the neighbourhood of dynamic phase transitions, which do not emerge in the thermodynamic behaviour of typical ferromagnets [20]. Keeping these facts mentioned above in mind, it is possible to say that more work is required to understand the origin of dynamic phase transitions of magnetic systems driven by oscillatory magnetic field.

*Corresponding Author: Department of Physics, Faculty of Science, Dokuz Eylul University, Izmir, Turkey, erol.vatansever@deu.edu.tr 
In addition to the works regarding the influences of an oscillating magnetic field (uniform over space) on the dynamic characteristics of the magnetic systems, some efforts were taken to investigate the dynamic features resulting from standing and/or propagating magnetic field waves [21-24]. It is possible to say that propagating magnetic field wave is an example of spatially and also of temporally changed magnetic field being exposed to a magnetic system. In Ref. [21], the authors applied their theoretical model to elucidate the dynamical modes and nonequilibrium phase transition of the spin-1 Blume-Capel model for two-dimensional square lattice, within the framework of MC simulation. It has been found that, the wavelength of the external field plays an important role in the dynamic nature of the considered system, in addition to the amplitude and period of the field. The system presents two dynamical phases: Propagating spin wave and spin frozen or pinned phases. In another interesting work, the physics behind a simple ferromagnet under the influence of a propagating magnetic field has been discussed in detail [22]. It is shown that dynamic phase boundary constructed in temperature versus applied field amplitude tends to shrink as the strength of the wavelength of the external field decreases. It is clear that particular attention in the works mentioned above has only been devoted to clarifying the physics in the bulk materials. To the best of our knowledge, there is no any attempt to address the same problem for nanoparticles with surfaces, especially for magnetic nanowires, driven by a propagating or (and) standing magnetic field wave(s). Magnetic nanowires are important materials and also potential candidates for applications in advanced nanotechnology including magnetic memory devices [25-26] and biomedical applications [27] due to their own distinctive magnetic properties. For the sake of completeness, we would like to emphasize that nonequilibrium dynamics as well as phase transition characteristics of various types of magnetic nanowire systems under the existence of an oscillating field (uniform over space) have been studied by employing Effective-field theory [28-34] and also MC simulation method [35-36]. From this point of view, we intend to investigate the dynamic behaviours of cylindrical nanowire system being subjected to a propagating magnetic field (variation in spatio-temporal), by utilizing MC simulation method. Our obtained results indicate that the present system exhibits unusual and interesting magnetic dynamics originating from the occurrence of the propagating magnetic field, which have not been observed and discussed so far, in view of the magnetic nanostructures.

The outline of the remainder parts of the paper is as follows: In section 2, we present the model and simulation details. The results and discussion are given in section 3, and finally section 4 includes our conclusions.

\section{FORMULATION}

The Hamiltonian of the spin-1/2 cylindrical nanowire (as shown in Fig. 1) driven by a propagating magnetic field can be written as follows:

$$
\widehat{H}=-J \sum_{\langle i j\rangle} S_{i}^{z}(x, y, z, t) S_{j}^{z}\left(x^{\prime}, y^{\prime}, z^{\prime}, t\right)-\sum_{i} h(x, y, z, t) S_{i}^{z}(x, y, z, t)
$$

here $S_{i}^{z}(x, y, z, t)= \pm 1$ is the Ising spin variable at any position $x, y$ and $z$ in the

nanowire. $J(>0)$ is the ferromagnetic spin-spin coupling term. The first summation in Eq. [1] is over the nearestneighbor spin couplings while the second one is over all lattice sites in the system. $h(x, y, z, t)$ represents the propagating magnetic field, which has the following form [21]:

$$
h(x, y, z, t)=h_{0} \cos (w t-k z)
$$

For the sake of simplicity, the magnetic field is propagating in the $z$ direction (long axis of the nanowire). It is obvious that it varies with time and position of the nanowire. Here, $h_{0}, w$ and $k$ refer to the amplitude, angular frequency and wave vector of the external field, and $t$ is time. Period and wavelength of the magnetic field are $\tau=2 \pi / w$ and $\lambda=2 \pi / k$, respectively. It may be noted here that we have fixed the period of the external field as $\tau=100$ throughout the study. 


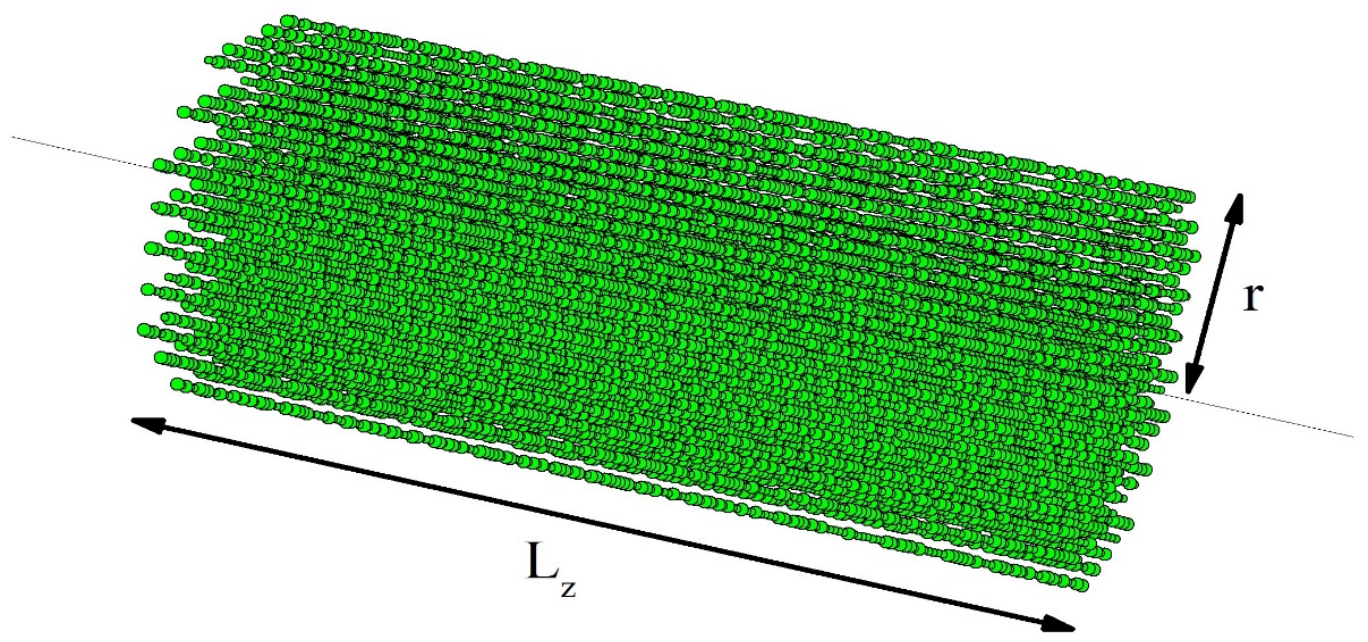

Figure 1. Schematic representation of the spin-1/2 Ising cylindrical nanowire with length $L_{z}$ and radius $r$.

By utilizing Metropolis algorithm [37-38], we employ MC simulation to understand the magnetic nature of the cylindrical nanowire system driven by a propagating magnetic field wave. The nanowire system is located on a simple cubic lattice, which has a length of $L_{z}=200$ (lattice space) and a radius $r=10$ (lattice space). For these selected values of the $L_{z}$ and $r$, total number of the spins in the system is $N_{t}=63400$. In order to mimic the cylindrical nanowire, we follow the boundary conditions such that it is free in $x y$ plane and periodic along the $z$ direction of the system. The simulation procedure we follow in this study can be briefly summarized as follows. The

We have calculated the instantaneous value of the magnetization at time $t$ as follows:

$$
M(t)=\frac{1}{N_{t}} \sum_{i=1}^{N_{t}} S_{i}^{z}(x, y, z, t) .
$$

Using $M(t)$, dynamic order parameter of the cylindrical nanowire system can be defined as follows:

$$
Q=\frac{1}{\tau} \oint M(t) d t
$$

In order to detect the dynamic phase transition points of the present system, in addition to the derivative of dynamic order parameter $d Q / d T$, we check the variance of the $Q$ and internal energy $(E)$, which are defined as follows:

$$
\chi_{Q}=N_{t}\left(\left\langle Q^{2}\right\rangle-\langle Q\rangle^{2}\right)
$$

and

$$
\chi_{E}=N_{t}\left(\left\langle E^{2}\right\rangle-\langle E\rangle^{2}\right)
$$

here $E$ is the internal energy given in Eq. [1] over a full cycle of the propagating magnetic field, which is defined in the following form:

$$
E=-\frac{1}{\tau N_{t}} \oint \widehat{H} d t
$$




\section{RESULTS AND DISCUSSION}

The microscopic spin configurations of the present cylindrical nanowire system show that the system demonstrates two different dynamical phases, which sensitively
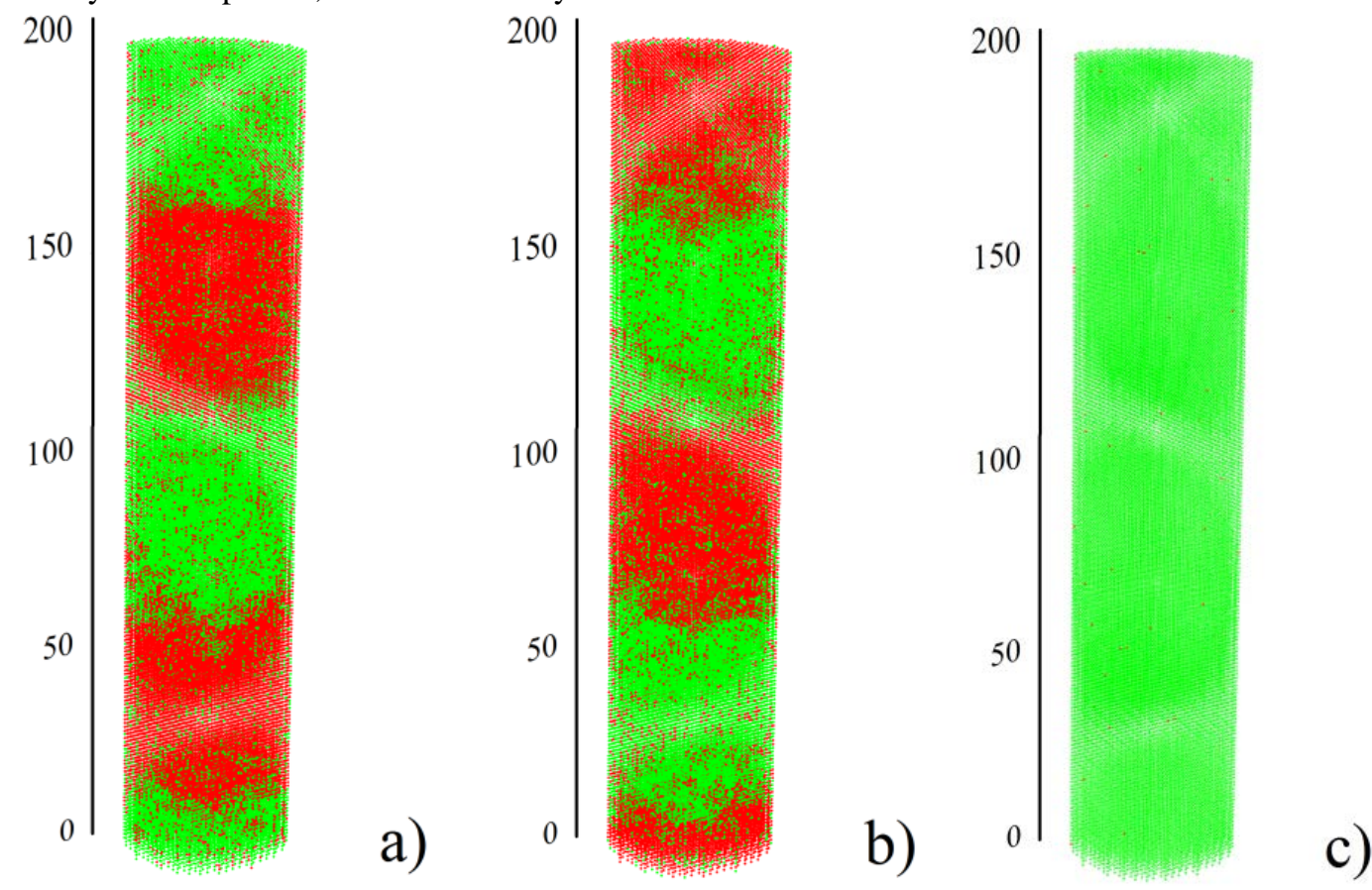

Figure 2. Coherent propagation of spin clusters of up (green dots) and down (red dots) spins, swept by a propagating magnetic field wave. The spin snapshots are taken for two different values of the Monte Carlo simulation time (a) $t=50100$ MCSS, (b) $t=50200$ MCSS for values of the reduced temperature $k_{B} T / J=4.0$, amplitude $h_{0} / J=0.3$, wavelength $\lambda=100$ and period $\tau=100$ of the external magnetic field. (c) corresponds to spin snapshot at time $t=50100$ MCSS, for the same parameters with (a) and (b), except from considered value of temperature $k_{B} T / J=1.5$.

Depend on the considered values of the system parameters. Two alternate bands of spin values $S= \pm 1$ are found, and they tend to propagate along the long axis of the nanowire (namely along $z$ axis of nanowire), in the high temperature regions. In Fig. 2, we can see easily the coherent propagation of the spin bands. These spin snapshots are taken for two different values of the MC simulation time (a) $t=50100$ MCSS, (b) $t=50200$ MCSS for values of the reduced temperature $k_{B} T / J=4.0$ and amplitude $h_{0} / J=0.3$ and wavelength $\lambda=100$ of the external magnetic field. The cylindrical nanowire includes two full waves for the considered value of $\lambda$. Our MC simulation results also suggest that a reduction in the value of temperature destroys the coherent propagating of spin bands of the cylindrical nanowire system, leading to a spin-frozen or pinned phase [21], as shown in Fig. 1(c) which is displayed for value of $k_{B} T / J=1.5$, with all other parameters of propagating magnetic field remain the same. At the lower temperature regions, ferromagnetic spin-spin coupling dominates against the propagating magnetic field, hence, most of the spins in the nanowire are frozen or pinned to any one value of $S$. It is clear that these microscopic behaviors sensitively depend on the studied system parameters. We should note that these types of coherent propagations of spin bands and also spin-frozen phases have been recently observed in bulk materials under the existence of a propagating magnetic field [2123].

In Figs. 3(a-d), we focus our attention on the effects of the varying field amplitudes on the thermal variations of the dynamic order parameter $(Q)$, its derivative $d Q / d T$, variance of $Q\left(\chi_{Q} / N_{t}\right)$ and of $E\left(\chi_{E} / N_{t}\right)$. The curves are depicted for three values of the applied field amplitudes, i.e., $h_{0} / J=0.1,0.3$ and 0.5 with $\tau=100$ and $\lambda=100$. As shown in Fig. 3(a), $Q$ gradually decreases starting from its saturation value with increasing thermal energy, and it vanishes continuously at the critical temperature. 

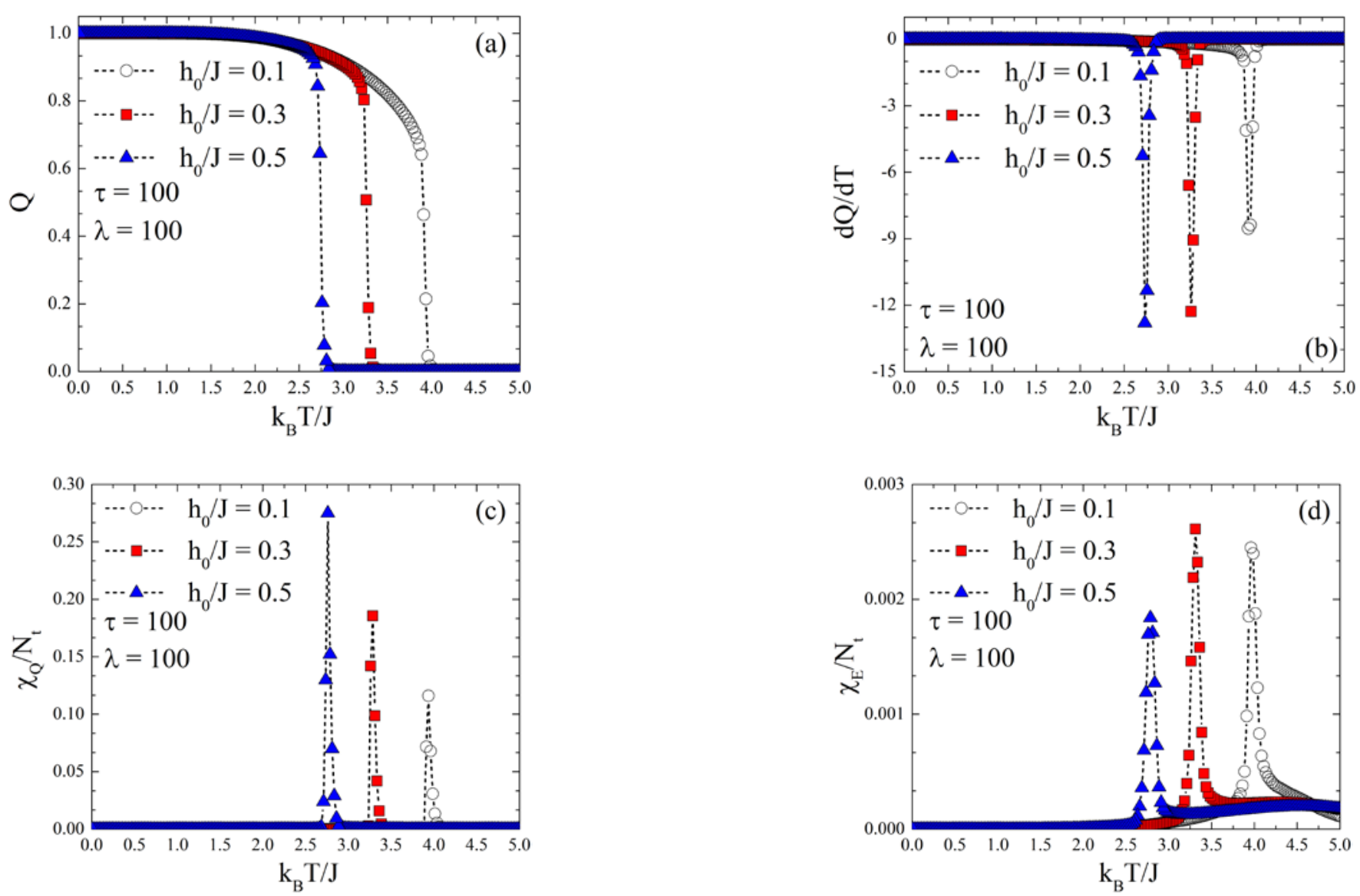

Figure 3. Thermal variations of the (a) dynamic order parameter (Q), (b) $d Q / d T$, (c) variance of $Q\left(\chi_{Q} / N_{t}\right)$ and (d) of $E$ $\left(\chi_{E} / N_{t}\right)$ for different values of reduced amplitude $h_{0} / J$ of the external field. The curves are depicted for values of $\tau=100$ and $\lambda=100$.

Dynamic phase transition point strongly depends on the component of the propagating magnetic field. Our MC findings underline that an increment in value of the applied field amplitude leads to a decrement in the location of phase transition point.

At the higher temperature regions, $Q$ is zero and this corresponds to a phase where the coherent propagation of spin bands exhibit. In the lower temperature regions, the system exhibits dynamically ferromagnetic phase, where $Q$ is nonzero.

As discussed earlier, the microscopic spin snapshots show that the system presents low temperature spin-frozen phase in these regions. Based on these spin configurations of the cylindrical nanowire system, it is possible to say that the system undergoes a dynamical phase transition from a coherent spin propagation phase to a spin-frozen phase, when the temperature is decreased starting from a relatively higher value. For a selected combination of the Hamiltonian parameters, the temperature dependencies of $d Q / d T$ show a sharp dip while $\chi_{Q}$ and $\chi_{E}$ reveal a very sharp peak indicating the existence of a second order phase transition, as depicted in Figs. 3(b-d). These peaks are found to shift to the lower temperature values when the strength of $h_{0} / J$ is increased.

In order to have a better understanding about the influences of the varying values of wavelength of the propagating field on the system, we give the thermal variations of $Q, d Q / d T, \chi_{Q}$ and also $\chi_{E}$ in Figs. 4(a-d). The curves are given for two values of the wavelengths of the field, i.e., $\lambda=50$ and 100 with $h_{0} / J=0.3$ and $\tau=100$. It is found that dynamic phase transition points depend on the selected value of $\lambda$ of the propagating magnetic field wave. 

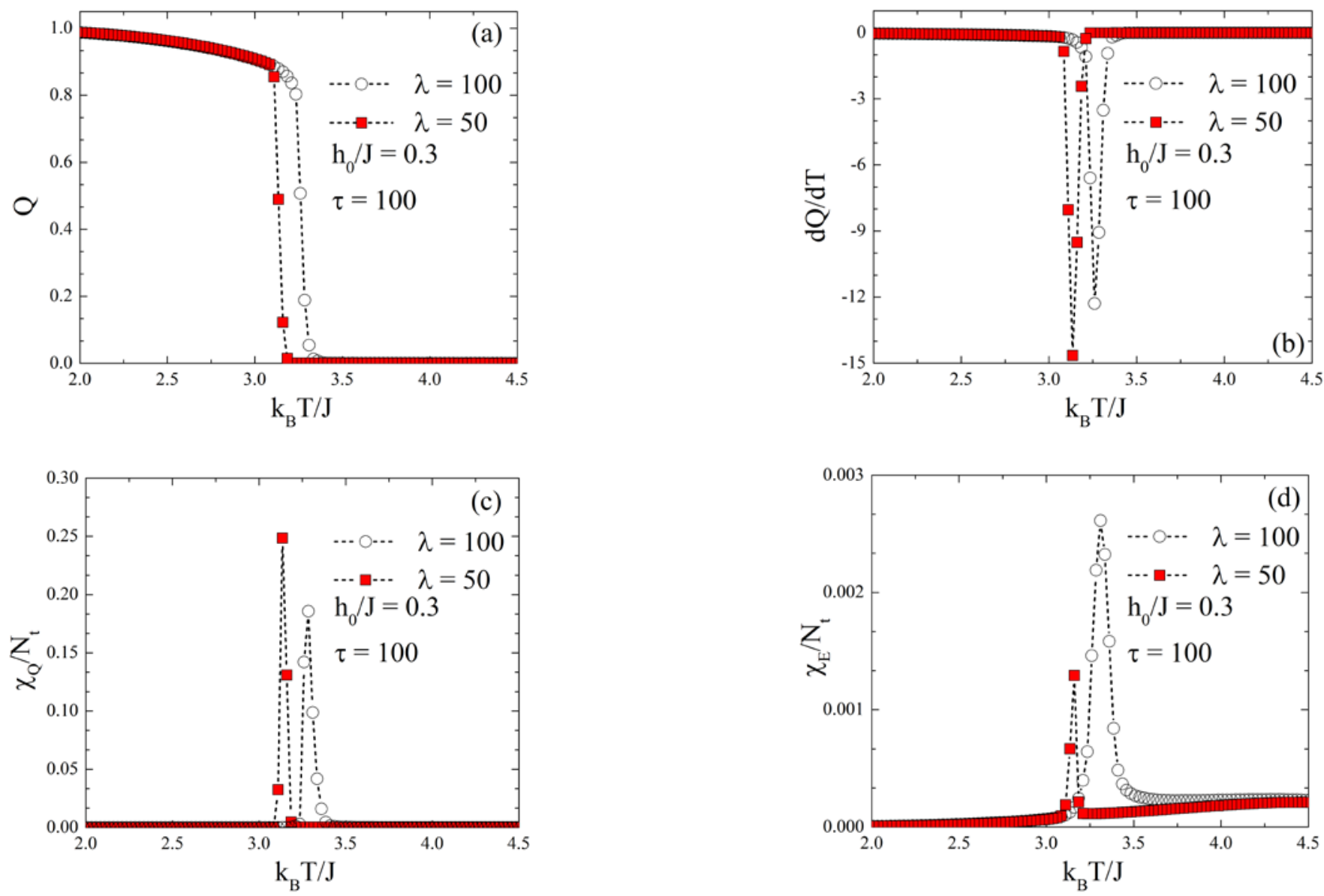

Figure 4. Temperature dependencies of the (a) $Q$, (b) $d Q / d T$, (c) $\chi_{Q} / N_{t}$ and (d) $\chi_{E} / N_{t}$ at various values of wavelength $\lambda$ of the external field. The curves are depicted for values of $\tau=100$ and $h_{0} / J=0.3$.

As seen in these figures, thermal variations of $Q, d Q / d T$, $\chi_{Q}$ and $\chi_{E}$ support that when the value of wavelength of the external field is increased, dynamically ferromagnetic phase region gets wider. It may be noted here that such types of observations originating from the variation of the wavelength of the propagating magnetic field have been found in Ref. [22], where kinetic Ising model on a 2D square lattice is exposed to a propagating magnetic field.

In order to elucidate the influences of the applied field amplitude on the dynamic phase transition features of the cylindrical nanowire system, we obtain the phase diagrams in $\left(k_{B} T_{C} / J-h_{0} / J\right)$ plane with two values of the wavelength of the propagating magnetic field with $\tau=100$, in figure 5 . We note that dynamic phase transition points are deduced from the peaks of the thermal variations of $d Q / d T, \chi_{Q}$ and $\chi_{E}$ curves. It is clear from the phase diagrams that when the applied field amplitude is increased, the dynamic phase transition points are shifted to the relatively lower temperature regions.

The aforementioned behaviors seem to be independent of the applied field wavelength $\lambda$. On the other hand, dynamic phase boundary line, which separates dynamically ordered phases from disordered phases, shrinks inward when the strength of the $\lambda$ of the external field decreases.

Recently, magnetic response of the spin-1/2 Ising cylindrical nanowire system to an oscillating magnetic field (uniform over space) has been investigated by means of MC simulation with Metropolis algorithm [35] for the same system parameters with the present study. 


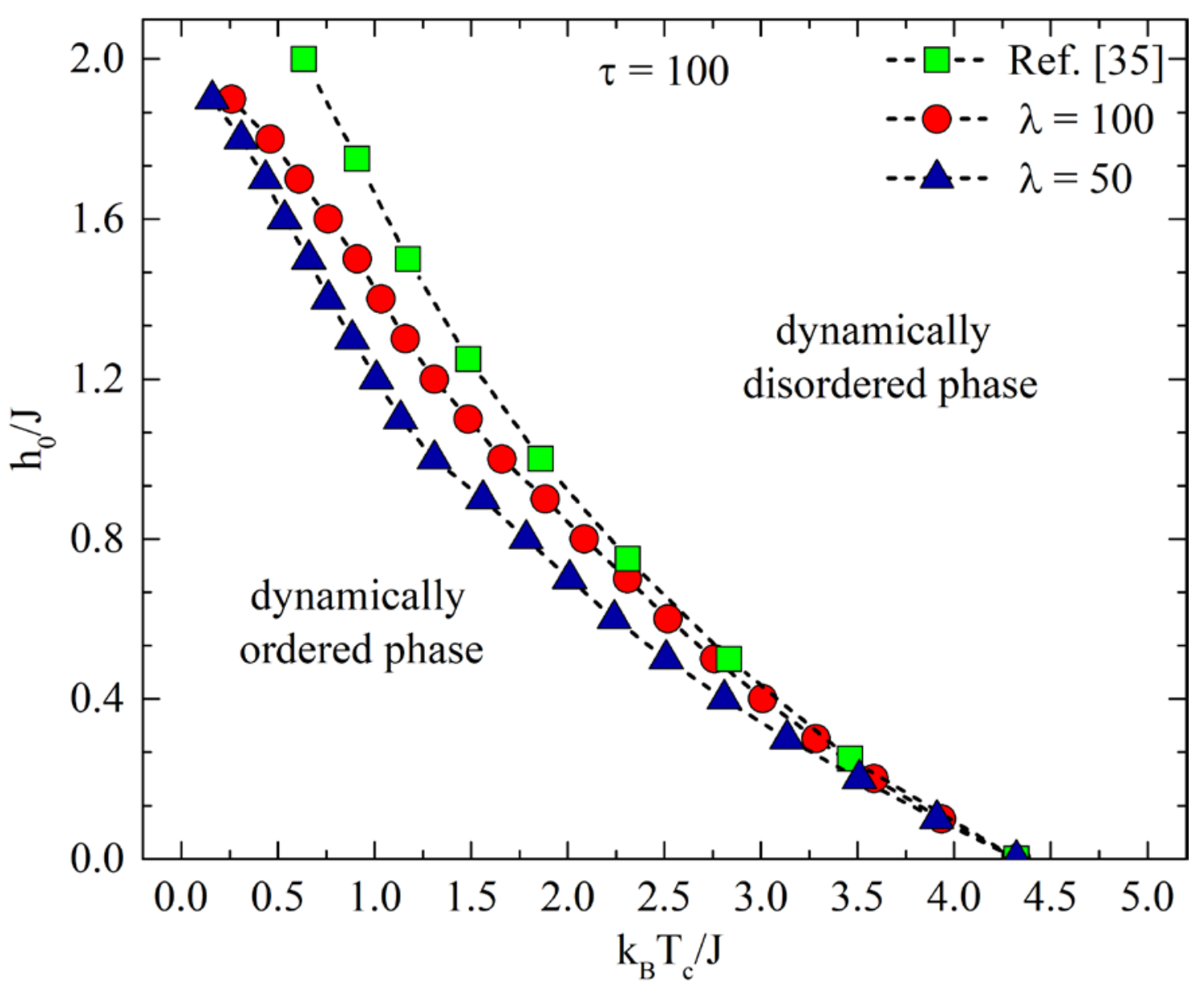

Figure 5. Dynamic phase diagram in the $\left(k_{B} T_{C} / J-h_{0} / J\right)$ plane for the cylindrical nanowire system under the presence of a propagating magnetic field. Different symbols correspond to the different values of applied field wavelengths: $\lambda=100$ (red bullet), and $\lambda=50$ (blue triangle). Here, green square symbols correspond to the phase transition points of the spin-1/2 cylindrical nanowire system under a time dependent oscillating magnetic field, which is uniform over space [35], for the same system parameters used in this study. Dynamic phase diagrams are given for value of $\tau=100$.

In order to make a comparison between propagating and oscillating magnetic fields, the results of the reference [35] are added to the Fig. 5 (green squares). It is obvious from the figure that there is no clear distinction between the magnetic field sources for the small values of the applied field amplitudes, in the sense of dynamic phase transition point. However, as the strength of $h_{0} / J$ is increased, dynamic phase boundaries begin to separate from each other. For considered values of the wavelengths of the propagating magnetic field in this study, our MC simulation findings indicate that phase transition points are always lower than those of the critical points obtained in reference [35], especially at the higher applied field amplitude regions. Note that in Ref. [40], the authors have studied the standing magnetic field effects on the spin- $1 / 2$ Ising model using Monte-Carlo simulations. They have also plotted the phase diagram in dynamic transition temperature and field amplitude for varying values of the wavelength. Even though there exists a clear difference between Ref. [40] and the current study, it may possible to compare the obtained phase diagrams. By comparing the
Fig. 6 of Ref. [40] with the Fig. 5 of the present study, it is possible to underline that when value of the wavelength is increased, dynamic phase transition temperature tends to shift to the upper transition regions.

In addition to the MC simulation studies, there are a few studies regarding the time dependent magnetic field effects on the spin-1/2 cylindirical nanowires, by means of Effective-Field Theory [28-34]. In these studies, external magnetic field is selected to be uniform over space.

It is possible to say that there are many similarities between the results in those found in previous studies and the current work. For example, for a fixed value of period of the external field, with increasing value of the applied field amplitude, dynamic phase transition points tend to shift to the lower temperature values. And also, it is possible to break the symmetry due to the applied field period. The aforementioned situations are also valid for both MC simulation and Effective-Field Theory methods. 


\section{CONCLUDING REMARKS}

To conclude, we study the nonequilibrium dynamics and phase transition features of the ferromagnetic spin-1/2 cylindrical nanowire under the existence of a propagating magnetic field. For this investigation, we use Monte Carlo simulation method with single-site update Metropolis algorithm. Based on the obtained results in this study, it is possible to mention that there are two types of dynamical states in the system, depending on the considered system parameters. The results can be summarized as follows: In the higher temperature regions, two alternate bands of spins are found, and they tend to coherently propagate along the long axis of the nanowire. However, a decrement in the value of temperature leads to the occurrence of spinfrozen or spin pinned phase. This phase generally occurs at the relatively lower temperature regions, as in the case of the conventional bulk systems under the influence of a propagating magnetic field [21-23].

Additionally, we give the dynamic phase diagram of the spin-1/2 cylindrical nanowire in $\left(k_{B} T_{C} / J-h_{0} / J\right)$ plane for two values of the wavelengths of the propagating magnetic field. Our MC simulation findings clearly indicate that as the strength of the field amplitude is increased, the phase transition points tend to shift to the relatively lower temperature regions. In the sense of wavelength of the field, it is found that dynamic phase boundary line shrinks inward in the related plane when the value of $\lambda$ of the external field decreases. We also observed that the dynamic behavior of the present system with a propagating magnetic field exhibits quite different characteristics in comparison with the same system, but only in the presence of a sinusoidal oscillating magnetic field (uniform over space) [35].

Very recently, it has been shown that frequency dispersion of the nanocubic core/shell particle driven by an oscillating field (uniform over space) can be categorized into three groups, as in the case of the conventional bulk systems [11]. Keeping these in mind, it would be interesting to investigate the influences of the propagating magnetic field on the frequency dispersion of dynamic loop area of the present system. From the theoretical perspective, the effects of standing magnetic field on the present system and also on the quenched disordered binary alloy cylindrical nanowire [39] could also be studied. We believe that such types of studies will be beneficial to provide deeper understanding of physics underlying of nanoscale materials driven by a time dependent magnetic field.

\section{ACKNOWLEDGEMENTS}

The author is thankful to Muktish Acharyya from Presidency University for valuable discussions and suggestions. The numerical calculations reported in this paper were performed at TÜBİTAK ULAKBIM (Turkish agency) High Performance and Grid Computing Center (TRUBA Resources).

\section{REFERENCES}

[1] T. Tomè and M.J. de Oliveira, "Dynamic phase transition in the kinetic Ising model under a time dependent oscillating magnetic field” Phys. Rev. A, vol. 41, pp. 4251-4254, 1990.

[2] W.S. Lo and R.A. Pelcovits, "Ising model in a time dependent magnetic field” Phys. Rev. A, vol. 42, pp. 74717474, 1990.

[3] S.W. Sides, P.A. Rikvold and M.A. Novotny, Phys. Rev. Lett. vol. 81, pp. 834-837 1998.

[4] G.M. Buendia and E. Machado, "Magnetic behaviour of a mixed Ising ferrimagnetic model in an oscillating magnetic field” Phys. Rev. B, vol. 61, pp. 14686-14690, 2000.

[5] G.M. Buendia and P.A. Rikvold, "Dynamic phase transition in the two-dimensional kinetic Ising model in an oscillating magnetic field: Universality with respect to the stochastic dynamics” Phys. Rev. E, vol. 78, pp. 051108 2008.

[6] B. Chakrabarti and M. Acharyya, "Dynamic transitions and hysteresis”Rev. Mod. Phys. vol. 71, pp. 847-859, 1999.

[7] M. Keskin, O. Canko and Ü. Temizer, "Dynamic phase transition in the kinetic spin-1 Blume-Capel model under a time dependent oscillating external field” Phys. Rev. E, vol. 72, pp. 0361252005.

[8] X. Shi, G. Wei and L. Li, "Effective-field theory on the kinetic Ising model” Phys. Lett. A, vol. 372, pp. 59225926, 2008.

[9] H. Park and M. Pleimling, "Surface criticality at a dynamic phase transition” Phys. Rev. Lett., vol. 109, pp. 175703, 2012.

[10] Y. Yüksel, E. Vatansever and H. Polat, "Dynamic phase transition properties and hysteretic behaviour of a ferrimagnetic core-shell nanoparticle in the presence of a time dependent magnetic field” J. Phys.: Condens. Matter, vol. 24, pp. 436004, 2012.

[11] E. Vatansever, "Monte Carlo simulation of dynamic phase transitions and frequency dispersions of hysteresis curves in core/shell ferrimagnetic cubic nanoparticle" Phys. Lett. A, vol. 381, pp. 1535-1542, 2017.

[12] E. Vatansever and H. Polat, "Dynamic phase transitions in a ferromagnetic thin film system: A Monte Carlo simulation study” Thin Solid Films, vol. 589, pp. 778-782, 2015.

[13] M. Acharyya, "Nonequilibrium phase transition in the kinetic Ising model: Divergences of fluctuations and responses near the transition point” Phys. Rev. E, vol. 56, pp. 1234, 1997.

[14] M. Acharyya, "Nonequilibrium phase transition in the kinetic Ising model: Critical slowing down and the specific-heat singularity” Phys. Rev. E, vol. 56, pp. 2407, 1997. 
[15] R.A. Gallardo, O. Idigoras, P. Landeros and A. Berger, "Mean field theory of dynamic phase transition in ferromagnets” Physica B, vol. 407, pp. 1377-1380, 2012.

[16] Y.-L. He and G.-C. Wang, "Observation of dynamic scaling of magnetic hysteresis in ultrathin ferromagnetic Fe/Au(001) films” Phys. Rev. Lett. vol. 70, pp. 2336-2339, 1993.

[17] D.T. Robb, Y.H. Xu, O. Hellwig, J. McCord, A. Berger, M.A. Novotny and P.A. Rikvold, "Evidence for a dynamic phase transition in $[\mathrm{Co} / \mathrm{Pt}] 3$ magnetic multilayers” Phys. Rev. B., vol. 78, pp. 1344222008.

[18] J.-S. Suen and J.L. Erskine, "Magnetic hysteresis dynamics: Thin $\mathrm{p}(1 \mathrm{X} 1)$ Fe films on flat and stepped W(110)” Phys. Rev. Lett., vol. 78, pp. 35671997.

[19] A. Berger, O. Idigoras and P. Vavassori, “Transient behaviour of the dynamically ordered phase in uniaxial cobalt films” Phys. Rev. Lett., vol. 111, pp. 190602, 2013.

[20] P. Riego, P. Vavassori and A. Berger, "Metamagnetic anomalies near dynamic phase transitions" Phys. Rev. Lett., vol. 118, pp. 1172022017.

[21] M. Acharyya and A. Halder, "Blume-Capel ferromagnet driven by propagating and standing magnetic field wave: Dynamical modes and nonequilibrium phase transition” J. Magn. Magn. Mater. vol. 426, pp. 53-59, 2017.

[22] M. Acharyya, "Polarized electromagnetic wave propagation through the ferromagnet: Phase boundary of dynamic phase transition”Acta Phys. Pol. B, vol. 45, pp. 1027-1036, 2014.

[23] M. Acharyya, "Dynamic symmetry breaking breathing and spreading transitions in ferromagnetic film irradiated by spherical electromagnetic wave” J. Magn. Magn. Mater., vol. 354, pp. 349-354, 2014.

[24] M. Acharyya, "Ising metamagnet driven by propagating magnetic field wave: Nonequilibrium phases and transitions” J. Magn. Magn. Mater., vol. 382, pp. 206210, 2015.

[25] M.I. Irshad, F. Ahmad and N.M. Mohamed, “A reviews on nanowires as an alternatives high density magnetic storage media” AIP Conf. Proc., vol. 1482, pp. 625-632, 2012.

[26] Y.P. Ivanov, A. Chuvilin, S. Lopatin and J. Kosel, "Modulated magnetic nanowires for controlling domain wall motion: Towards 3D magnetic memories” ACS Nano, vol. 10, pp. 5326, 2016.

[27] Y.P. Ivanov, A. Alfadhel, M. Alnassar, J.E. Perez, M. Vazquez, A. Chuvilin and J. Kosel, "Tunable magnetic nanowires for biomedical and harsh environment applications” J. Sci. Rep., vol. 6, pp. 24189, 2016. [28] B. Deviren, E. Kantar and M. Keskin, "Dynamic phase transition in cylindrical Ising nanowire under a time dependent oscillating magnetic field” J. Magn. Magn. Mater., vol. 324, pp. 2163-2170, 2012.

[29] B. Deviren, M. Ertaş and M. Keskin, "Dynamic magnetizations and dynamic phase transitions in a transverse cylindrical Ising nanowire” Phys. Scr., vol. 85, pp. 055001, 2012.

[30] E. Kantar, B. Deviren and M. Keskin, "Magnetic properties of mixed Ising nanoparticles with core-shell structure” Eur. Phys. J. B., vol. 86, pp. 253, 2013.

[31] M. Ertaş and Y. Kocakaplan, "Dynamic behaviors of the hexagonal Ising nanowire” Phys. Lett. A, vol. 378, pp. 845-850, 2014.

[32] B. Deviren and M. Keskin, "Thermal behaviour of dynamic magnetizations, hysteresis loop areas and correlations of a cylindrical Ising nanotube in an oscillating magnetic field within the effective field theory and the Glauber type stochastic dynamic approach” Phys. Lett. A, vol. 376, pp. 1011-1019, 2012.

[33] B. Deviren, Y. Sener, M. Keskin, "Dynamic magnetic properties of the kinetic cylindrical Ising nanotube" Physica A, vol. 392, pp. 3969-3983, 2013.

[34] E. Kantar, M. Ertaş and M. Keskin, "Dynamic phase diagrams of a cylindrical nanowire in the presence of a time dependent magnetic field” J. Magn. Magn. Mater., vol. 361, pp. 61, 2014.

[35] Y. Yüksel, "Monte Carlo study of magnetization dynamics in uniaxial ferromagnetic nanowires in the presence of oscillating and biased magnetic fields" Phys. Rev. E, vol. 91, pp. 032149, 2015.

[36] Y. Yüksel, "Dynamic phase transition phenomena and magnetization reversal process in uniaxial ferromagnetic nanowires” J. Magn. Magn. Mater., vol. 389, pp. 34-39, 2015.

[37] K. Binder, Monte Carlo Methods in Statistical Physics, Springer, Berlin, 1979.

[38] M.E.J. Newman and G.T. Barkema, Monte Carlo Methods in Statistical Physics, Clarendon Press, Oxford, 2001.

[39] Z.D. Vatansever and E. Vatansever, "Finite temperature magnetic phase transition features of the quenched disordered binary alloy cylindrical nanowire” J. Alloys Compd. vol. 701, pp. 288, 2017.

[40] A. Halder and M. Acharyya, "Standing magnetic wave on Ising ferromagnet: Nonequilibrium phase transition” J. Magn. Magn. Mater., vol. 420, pp. 290, 2016. 